\title{
Numerical Modeling of D-Mapping with Applications to Chemical Kinetics
}

S.K. Dey 
NASA Technical Memorandum 84332

\section{Numerical Modeling of D-Mapping with Applications to Chemical Kinetics}

S. K. Dey, Ames Research Center, Moffett Field, Calıfornıa

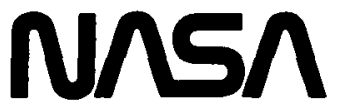

Natıonal Aeronautıcs and

Space Administration 
NUMERICAL MODELING OF D-MAPPINGS WITH APPLICATIONS TO CHEMICAL KINETICS

\title{
S. R. Dey
}

Computational Fluld Dynamics Branch, NASA Ames Research Centex, Moffett Field, California 94035

\begin{abstract}
Numerical modeling of D-mappings has been studied and applied to solving nonlinear stiff systems. These mappings have been locally ifnearlzed for convergence analysis, and some applications have been made to chemical
\end{abstract} kinetics.

Keywords. Nonlinear equations; numerical methods; blomedical.

\section{INTRODUCTION}

A numerical method was developed by Dey (1977) for solving nonlinear systems, some applications of which were later made to stiff syscems (Dey, 1982). Convergence analysis was done using nonlinear D-mappings (Dey, 1981).

It is extremely difficult to represent this analysis computationally. Local linearization for such an analysis, which rendered computational modeling of $D$-mappings feasible, was suggested by Lomax (1983). In this article we discuss linearized modeling of D-mappings and some applications of the method.

\section{D-MATRICES AND D-MAPPINGS}

If a sequence of square matrices of the same order satisfy the following condition,

$$
\lim _{k \rightarrow \infty} A_{k} A_{k-1} \cdot \cdot A_{1}=0
$$

each $A_{k}$ is called a D-matrix. A D-matrix is not necessarily a convergent matrix, and conversely.

Theorem 1. A sufficient condition that $A_{k}$ is a D-matrix is that

$$
\left\|A_{k}\right\|_{q} \leq a<1
$$

$\forall k>k$ and that $q$ is the same $\forall k$.

Theorem 1 is easily proved. Let

$$
\begin{aligned}
& u^{k}=\left(u_{1}^{k} u_{2}^{k} \cdot u_{J}^{k}\right)^{T} \in D_{k} \text {, } \\
& k=1,2, \ldots\left(u_{j}^{k}=\text { value of } u_{j}\right. \text { at some } \\
& \text { kth iteration). } \\
& \text { Let us consider a chained linear spaces }
\end{aligned}
$$

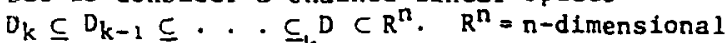

$$
\begin{aligned}
& \text { real space. Let } u^{k}, u^{*} \in D_{k} \forall k \text { and }
\end{aligned}
$$

$$
\begin{aligned}
& G_{k}: D_{k+1} \times D_{k} \rightarrow D_{k+1} . \text { If } \\
& G_{k}\left(u^{k+1}, u^{k}\right)-G_{k}\left(u^{*}, u^{*}\right) \\
& \quad=A_{k}\left(u^{k+1}-u^{*}\right)+B_{k}\left(u^{k}-u^{*}\right)
\end{aligned}
$$

and $\forall k>K$, and if $\left(I-A_{k}\right)^{-1} B_{k}$ is a D-matrix, $G_{k}$ is called a D-mapping (Dey, 1981).

If we now consider a nonstationary iterative scheme of the form

$$
u^{k+1}=G_{k}\left(u^{k+1}, u^{k}\right)
$$

and $1 f \quad G_{k} \cdot D_{k+1} \times D_{k} \rightarrow D_{k+1}$ is a D-mapping, then

$$
\lim _{k \rightarrow \infty} u^{k}=u^{\star}
$$

where $u^{\star}=G_{k}\left(u^{\star}, u^{\star}\right) \forall k$ (Dey, 1981).

\section{IOCALLY LINEARIZED D-MAPPING}

Let us Inearize (4) on $D_{k} \times D_{k}$, using first-order approximation of $G_{k}\left(u^{k+1}, u^{k}\right)$ near $\left(u^{k}, u^{k}\right)$. Then,

$$
u^{k+1}=G_{k}\left(u^{k}, u^{k}\right)+G_{k}^{\prime}\left(u^{k+1}-u^{k}\right)
$$

where $G_{k}^{\prime}$ is the Fréchet derivative of $G_{k}$ on $D_{k} \times D_{k}$. Equation (6) may be expressed as

$$
u^{k+1}=A_{k} u^{k}+b_{k}
$$

where $A_{k}=-\left(I-G_{k}^{\prime}\right)^{-1} G_{k}^{\prime}$,

$b_{k}=\left(I-G_{k}^{\prime}\right)^{-1} G_{k}\left(u^{k}, u^{k}\right)$. We have assumed that (I - $G_{k}^{i}$ ) is invertible. Now we may prove a second theorem. 
Theorem 2. If (1) $\left|A_{j}-A^{*}\right|<E$, where $E$ is a matrix consisting of elements that are positive and arbitrarily small and

(11) $\left|b_{j}-b^{*}\right|<\varepsilon, E$ is a vector consisting of elements that are positive and arbitrarily sma11, then (5) is true (convergence) if $A_{k}$ is a D-matrix (Dey, 1983a).

Theorem 3. If $G_{k}^{\prime}$ is a D-matrix, so is $A_{k}$ (Dey, 1983a).

This principle may now be applied computationally.

\section{PERTURBED FUNCTIONAL ITERATION}

Let a nonlinear system be expressed as

$$
\begin{aligned}
& u=G_{o}(u) \\
& u \in D, \quad G_{o}: D \rightarrow D
\end{aligned}
$$

A Gauss-Seldel-type 1teration for the solu$t$ ion may be expressed as

$$
\begin{aligned}
& u^{k+1}=G\left(u^{k+1}, u^{k}\right) \\
& G: D \times D+D, u^{k} \in D \forall k
\end{aligned}
$$

A perturbed fterative scheme (Dey, 1977) may be expressed as (in the element form)

$$
u_{j}^{k+1}=w_{j}^{k+1}+G_{j}\left(u^{k+1}, u^{k}\right)
$$

where

$$
\begin{aligned}
w_{j}^{k+1}= & {\left[G_{j}\left(G_{j}^{k+1, k}\right)-G_{j}^{k+1, k}\right] } \\
& \times\left[1-\partial_{j} G_{j}^{k+1, k_{j}^{-1}}(11 a)\right. \\
G_{j}^{k+1, k} & =G_{j}\left(u_{1}^{k+1} \cdot \cdots \cdot u_{j-1}^{k+1}, u_{j}^{k} \cdot \cdots u_{J}^{k}\right)
\end{aligned}
$$

$$
\begin{aligned}
G_{j}\left(G_{j}^{k+1, k}\right)=G_{j}\left(u_{1}^{k+1} \cdot \cdot u_{j-1}^{k+1},\right. \\
\left.\quad G_{j}^{k+1, k}, u_{j+1}^{k} \cdot \cdots \cdot u_{j}^{k}\right)
\end{aligned}
$$

$$
\begin{aligned}
& \partial_{J}\left(G_{J}^{k+1, k}\right)=\left[\partial G_{j} / \partial u_{J}\right]_{u_{1}^{k+1}, u_{2}^{k+2}} . \\
& u_{j-1}^{k+1}, G^{k+1, k} \text {, } \\
& u_{j+1}^{k} \cdot \cdot u_{J}^{k}
\end{aligned}
$$

The $\omega_{3}$ term 15 a perturbation parameter which accelerates the rate of convergence of (9) and stabilizes the numerical algorithm.

It has been proved (Dey, 1981) that if $G$ is a D-mapping on $D_{k+1} \times D_{k}$, a necessary and sufficlent condition for convergence is

$$
\lim _{k \rightarrow \infty}\left|\omega_{j}^{k}\right|=0 \mathrm{j}
$$

Following Theorems 2 and 3 we may prove that if $G_{k}$ is a D-matrix, then (12) is true (linearlzed sense). Recent results (Dey, 1983b) using local linearization indicates that if

$$
\max _{j, \mathrm{~m}}\left|G_{j \mathrm{~m}}\right| \leq B / J
$$

where $G_{f m}=\partial G_{j} / \partial u_{m}$ and $0 \leq \beta<1, G$ in (9) is a $\frac{\mathrm{D}}{\mathrm{C}}$-mapping. In order that (13) may be correct, certain input parameters for the system (e.g., mesh size and time-step) have to be chosen in special ways. If this cannot be found a convex-type operation may be defined as follows:

$$
\hat{G}_{J}(u, u)=\left(1-a_{j}\right) u_{j}+a_{J} G_{1}(u, u)
$$

Assuming $G_{j,}(u, u) \neq 1$, it has been found that $\hat{G}$ is a D-mapping (locally linearized) for the following:

$$
\begin{aligned}
& \text { 1. } \alpha_{j}=(-1)^{P}\left(1-G_{j \jmath}\right)^{-1} \text { if } G_{j m}=0 \text {, } \\
& m \neq j \text { and } P=0 \text { if } G_{j J}<1, P=1 \text { if } \\
& G_{j j}>1 \text {. } \\
& \text { 2. } \alpha_{j}=\min _{\substack{1 \leq m \leq J \\
m \neq j}}\left(\frac{\beta / J}{\left|G_{j m}\right|},(-1)^{p} \frac{(1+\beta / J)}{1-G_{J j}}\right)
\end{aligned}
$$

if $G_{j m} \neq 0$, m $\neq j$ and $p=0$ if $G_{j J}<1$, $p=l$ if $G_{j J}>1$.

where $\beta$ is such that

$$
\begin{aligned}
& 1>B \geq J\left(1+\frac{\left|1-G_{j \jmath}\right|}{\left|G_{\jmath \mu l}\right|}\right)^{-1} \\
& \text { 3. } \alpha_{J}=1 \text { If (13) Is satısfied. }
\end{aligned}
$$

The algorithm of perturbed functional iteration including a linearized convergence analys is may be briefly expressed as follows. At each iteration level, compute $G$ m. $m=1,2, \ldots ., J$. If (13) is satisfzed, set $\alpha_{1}=1$; otherwise, compute $\alpha_{j}$ using (15). If $\alpha_{j} \neq 1$, replace $G_{1}$ by $\hat{G}_{j}$, as given by (14). Compute w using (1la)-(Ild) and compute $u_{1}$ at the new iteration level by (10). If (12) is satisfied at some iteration level, convergence is found; if $G_{3 j}=1$, the method fails.

In general, for a $J \times J$ system the method requires ( 1 ) $\mathrm{J}^{2}+\mathrm{J}$ functionals to be computed for convergence analysis, (1i) partial linearization along the diagonal, and (121) no Jacobians.

It has been proved analytically (Dey, 1977) that in the vicinity of the root, the method should display a superlinear rate of convergence. 


\section{A DEGENERATE IMPLICIT CODE}

Let a nonlinear model be represented by

$$
\mathrm{du} / \mathrm{dt}=\mathrm{f}(\mathrm{u}), \quad \mathrm{u}=\left(\mathrm{u}_{2} \mathrm{u}_{2} \cdot \cdot \cdot \mathrm{u}_{\mathrm{J}}\right)^{\mathrm{T}}
$$

$u(0)=u_{0}$ (initial condition). Approximating (16) by a two-point backward-difference scheme, we get:

$$
u_{j}^{n+1}=u_{j}^{n}+\Delta t f_{j}\left(u^{n+1}\right), \Delta t=t i m e-s t e p
$$

This nonlinear system may now be solved by the above method which forms a degenerate implicit code (since the one-step, matrixinversion principle is not used for solution). If a convex-type operation of the form (14) is used, (16) becomes

$$
\mathrm{du} / \mathrm{dt}=(I-\alpha) \mathrm{du} / \mathrm{dt}+\alpha \mathrm{f}(\mathrm{u})
$$

where $\alpha=\operatorname{diag}\left(\alpha_{1}, \alpha_{2}, . \cdot \alpha_{J}\right)$ and $I=$ the identity matrix. If (16) is a stiff system, (condition number of $f^{\prime}(u) \gg 1$ ), it generally requires $\Delta t$ to be very small if a functional 1teration of the form (9) is applied for solution. In (18), $\alpha$ scales the elements in the Jacobian matrix $f^{\prime}(u)$, and using (15) for $a_{j}$ 's mean (for a given $\Delta t$ ), $D$-mapping is found so that perturbed functlonal iteration converges.

\section{APPLICATIONS}

\section{Application 1.}

(Bu1, 1979) $\dot{\mathrm{u}}_{1}=-10,004 \mathrm{u}_{1}+10,000 \mathrm{u}_{2}^{4}$; $\stackrel{\circ}{u}_{2}=u_{1}-u_{2}-u_{2}^{4} ; u_{1}(0)=u_{2}(0)=1$. An approximate solution is

$u_{2}(t)=i 10,004 \exp (-3 t) /[10,008$

$u_{1}(t)=(10,000 / 10,004) u_{2}^{4}$. As $t \rightarrow \infty, u_{1}(t) \rightarrow 0$, $u_{2}(t) \rightarrow 0$. Linearizing this system near $u_{1}(0), u_{2}(0)$, the condition number is $10^{4}$.

Using linearized convergence analysis for the degenerate Implicit code we got $\Delta t \cong 10^{-5}$, a sufficient condition for convergence if $\alpha_{3}=1$. Introducing (18) and computing $\alpha_{3}$ 's in a subroutine using (15) we used $\Delta t=10^{-3}$ to $10^{8}$; correct results were found. No program interruption was cased. Detalls may be found in Dey (1983b).

\section{Application 2. Irradiation of Neutral Water}

The model developed by Chacterjee and Magee and the analysis of its numerical solucion are given in Chatterjee and others (1983). The equations and the rate constants are given in Table 1 . For our present analysis we linearlzed the system and computed $a_{j}$ 's. Stiffness was measured by Strate (1983) at $\tau=0,0.1,1$, and 10 . Condition numbers are, respectively, $10^{19}, 10^{12}, 10^{12}, 10^{10}$ (approximately). This may be seen to be true in

Fig. 1. This pattem of solution was ana- lyzed by Chatterjee and Magee and was found to be valid. Here, difference equations were formed by approximating the derivatives by using the two-polnt trapezoidal rule. D-mappings were introduced, and time-accurate solutions were computed with $\Delta t=10^{-8}, 10^{-6}$.

\section{CONCLUSION}

Numerical solutions of stiff systems are generally obcalned by using multistep implicit codes (Miranker, 1981) whuch require inversion of matrices obtained by computing Jacobians. This has been avolded in the technique explained here. However, the code is dependent on the Jacoblans for its convergence analysis Such a linearized analysis seems to be quite effective, and, in contrast with its nonlinear counterpart, the complete analysis can be done computationally. More applications are under consideration.

\section{ACKNOWL EDGMENT}

I thank Il-year-old son Charlie Dey for writing codes for the first probled in TRS-80 color computer.

\section{REFERENCES}

Bu1, T. D. (1979). Some A-stable and L-stable methods for the numerical integration of stiff ordinary differential equations. J. ACM, 20, 3 .

Chatterjee, A., J. L. Magee, and S. K. Dey (1983). The role of homogeneous reactions in the radiolysis of water. Radiat. Res. (to appear).

Dey, S. K. (1977). Perturbed fterative solution of nonlinear equations with applications to fluid dynamics. J. Comp. Appl. Math. , 3, 1, 15-30.

Dey, S. K. (1981). Convergence analysis of a perturbed iterative scheme for solution of nonlinear systems, Int. J. Math $\underline{\mathrm{Sc1}} ., \underline{4}, 2,383-391$

Dey, S. K. (1982). Applications of perturbed functional iterations to nonlinear stiff initial value chemical kinetic problems. Proceedings of the Intemational Conference on Stiff Computations, Park City, Utah, Apri1 12-14.

Dey, S. K. (1983a). Numerical modelıng of linearized D-mappings with applications to stiff systems. NASA TM (to be published).

Dey, S. K. (1983b) Simulations of D-mappings in Elow models. Proceedings of Modeling and Simulation on Microcomputers. 83 Society of Computer Simulation, San Diego, Calif., Jan. 27-30

Lomax, H. (1983). NASA Ames Research Center, private communication.

Mtranker, W. L (1981) Numerical Merhods for Stiff Equations D. Reidel Publ. Co. Boston.

Strate, G. (1983). NASA Ames Research Center, private communication. 
Table 1 Differential Equations for Transient Species and Radiation Products in Irradiated Neutral Water

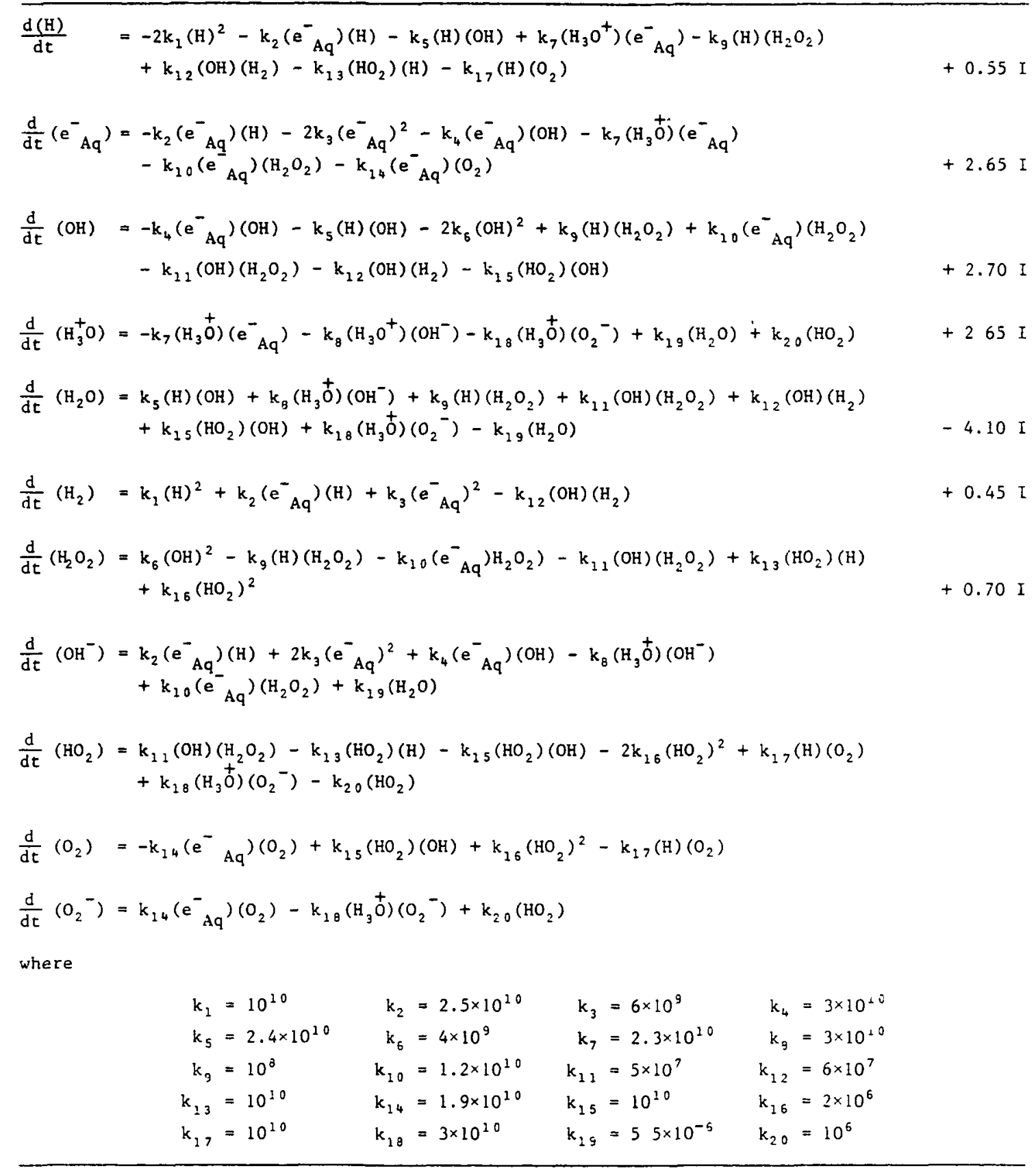




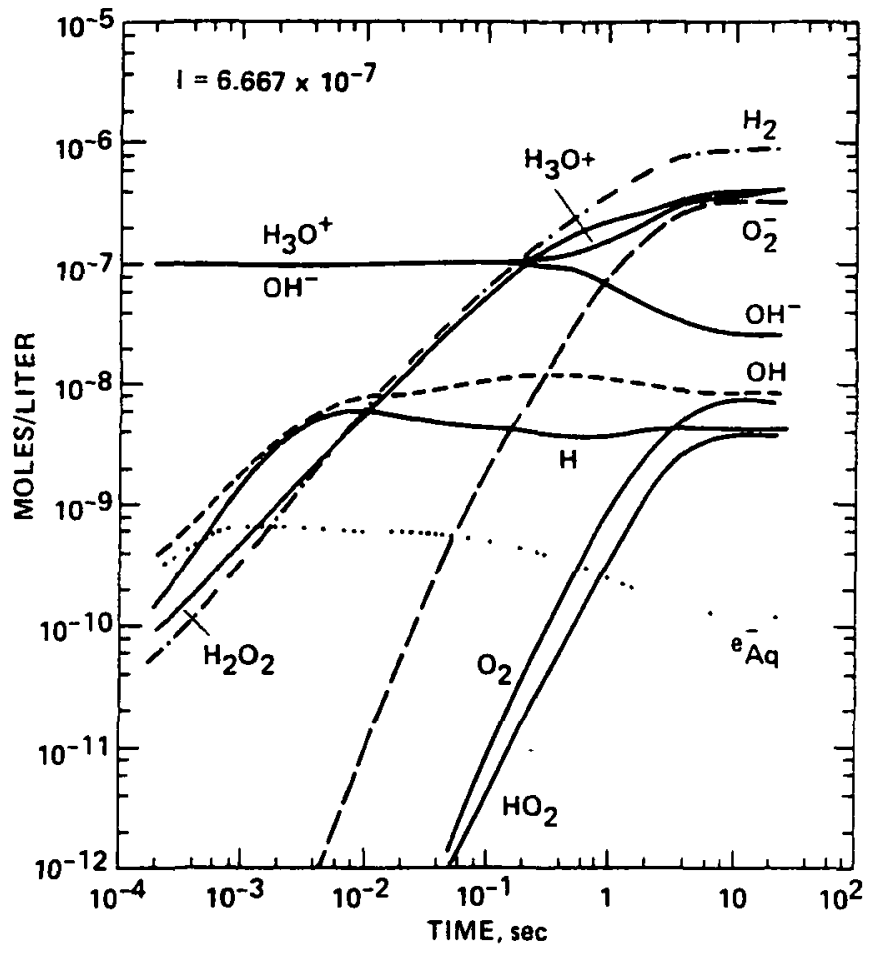

E1g. I Concentrations of species (Ex 2) vs. time for $I=6.667 \times 10^{-7}$ in the logarithmic scale up to $t=30 \mathrm{sec}$. (Here steady state is reached for all the species.) 


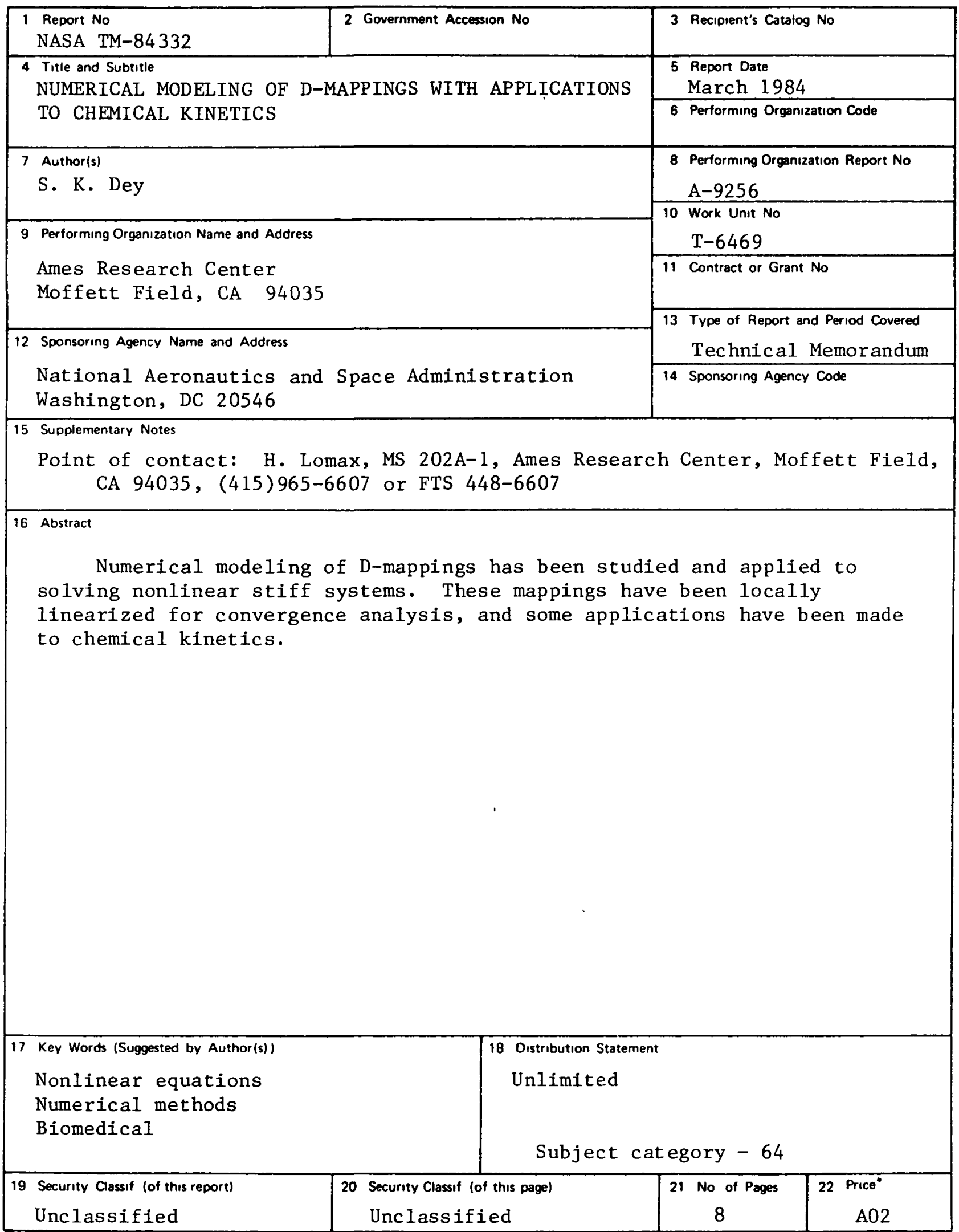

- For sale by the Natıonal Technical Information Service, Springfield, Virginia 22161 